Proyecciones

Vol. 20, Nº 3, pp. 323-337, December 2001.

Universidad Católica del Norte

Antofagasta - Chile

\title{
ON THE REPRESENTATION TYPE OF CERTAIN TRIVIAL EXTENSIONS
}

\author{
CRISTIÁN NOVOA \\ Universidade Católica de Goiás, Brasil \\ and \\ JOSÉ A. de la PEÑA \\ Universidad Nacional Autónoma de México, México
}

\begin{abstract}
Let $A \cong k Q / I$ be a basic and connected finite dimension algebra over closed field $k$. In this note show that in case $B=$ $A[M]$ is a tame one-point extension of a tame concealed algebra $A$ by an indecomposable module $M$, then the trivial extension $T(B)=B \propto D B$ is tame if and only if the module $M$ is regular.
\end{abstract}




\section{Introduction.}

Throughout this paper, $k$ denotes an algebraically closed field. By algebra $A$, we mean always a basic, connected and finite dimensional algebra over $k$ ( associative with 1 ). We denote by $\bmod A$ the category of finitely generated right $A$-modules, and $\mathcal{D}^{b}(A)$ the derived category of bounded complexs over the abelian category $\bmod A$ (see $[\mathrm{H}])$.

The concept of repetitive algebra was introduced by Hughes Waschbush $([\mathrm{HW}])$ in 1983, where their main interest was to obtain the classification of the finite representation self-injective algebras. In section 2 we recall some known facts about repetitive algebra. In this note, we will use the properties of repetitive categories to study the representation type of the trivial extension $T(B)=B \propto D B$, where $B$ is a one-point extension of a tame concealed algebra by an indecomposable module.

In section 3 we establish our main theorem on the representation type of the trivial extension $T(B)$. For that purpose, we prove that there exist a strong relation between the trivial extension $T(B)$ and the class of clannish algebras introduced by Crawley-Boevey in [C-B]. As a consequence of our main theorem we show that all tree algebra with non-negative Euler form $\chi_{A}$ of corank $\chi_{A} \leq 2$, have trivial extension of tame representation type.

This paper was done during a postdoctoral stay of the first named author at UNAM. Both authors thankfully acknowledge the finantial support of UNAM and CONACyT, México.

\section{Preliminaries.}

We recall that a quiver $Q=\left(Q_{0}, Q_{1}\right)$ is an oriented graph, where $Q_{0}$ is the set of vertices and $Q_{1}$ is the set of arrows. The ordinary quiver associated to an algebra $A$ will be denote by $Q_{A}$. The $k$-algebra $A$ will be called triangular when $Q_{A}$ has no oriented cycles. For each vertex $i$ of $Q_{A}$, we shall denote by $e_{i}$ the corresponding primitive idempotent of $A$, and by $S_{i}$ the corresponding simple $A$-module. We denote $P_{i}$ (respectively $I_{i}$ ) the projective cover (respectively, the injective envelope) of $S_{i}$. A bound quiver algebra $A \cong k Q / I$ will sometimes be considered as a $k$-category. 
Let $\mathcal{H}$ be a Krull-Schmidt category. By definition, the quiver $\Gamma(\mathcal{H})$ of $\mathcal{H}$ has as vertices the isomorphism classes $[M]$ of indecomposable objects $M \in \mathcal{H}$, and there are many arrows $[M] \longrightarrow[N]$ as the dimension of the space of irreducible maps from $M$ to $N$ in $\mathcal{H}$ (see $V I I .1$ [ARS]). If $\mathcal{H}=\bmod A$ or $\mathcal{D}^{b}(A)$, then $\Gamma(\mathcal{H})$ is a translation quiver (see 2.1 in $[R])$. The quiver $\Gamma(\bmod A)$, or $\Gamma_{A}$, is called the AuslanderReiten quiver of $A$. A translation quiver $\Gamma$ is called a tube (see VIII.4 in $[\mathrm{ARS}]$ ), if it contains cyclic paths and its topological realization is $|\Gamma|=S^{1} \times \mathbf{R}_{0}^{+}$(where $S^{1}$ is the unit circle and $\mathbf{R}_{0}^{+}$is the set of nonnegative real numbers). A $k$-category $A$ is called $\widetilde{\mathbf{A}}$-free whenever it contains no full sub category $B \cong k Q$ where the underlying graph of $Q$ is $\widetilde{\mathbf{A}_{\mathbf{n}}}$, for some $n$.

For the basic definitions and results of tilting theory, we refer the reader to [A1]. Two finite-dimensional $k$-algebras $A$ and $B$ are called tilting-cotilting equivalent, if there exist a sequence of algebras $A=$ $A_{0}, A_{1}, \ldots, A_{m+1}=B$ and a sequence of modules $T_{A}^{i}(0 \leq i \leq m)$ such that $A_{i+1}=E n d T_{A_{i}}^{i}$ and $T_{A_{i}}^{i}$ is either a tilting or cotilting module (see [A1]).

The one-point extension (respectively, coextension) of an algebra $A$ by an $A$-module $M$ will be denoted by $A[M]$ (respectively, $[M] A$ ). Let $A$ be a triangular algebra and $i$ a sink in $Q_{A}$. The reflection $S_{i}^{+} A$ (see $[\mathrm{HW}]$ ), of $A$ is defined as the quotient of the one-point extension $A\left[I_{i}\right]$ by the bilateral ideal generated by $e_{i}$. Dually, starting with a source $j$, we define the reflection $S_{j}^{-} A$.

By a polynomial-growth critical algebra, shortly pg-critical algebra (see 3 in $[\mathrm{NS}]$ ) we mean an alebra $A$ satisfying the following conditions:

1) $A$ or $A^{o p}$ is of one of the following form:

$$
C[M]=\left[\begin{array}{cc}
k & M \\
0 & C
\end{array}\right], \text { or } \quad C[N, t]=\left[\begin{array}{ccccccc}
k & k & \ldots & k & k & k & N \\
& k & \ldots & k & k & k & 0 \\
& & \ddots & \vdots & \vdots & \vdots & \vdots \\
& & & k & k & k & 0 \\
& & & & k & 0 & 0 \\
0 & & & & & k & 0 \\
& & & & & & C \\
& & & & & &
\end{array}\right]
$$


where $C$ is a representation infinite tilted algebra of type $\widetilde{\mathbf{D}_{\mathbf{n}}}$ with $(4 \leq n)$, with a complete slice in the preinjective component, and $M$ (respectively, $N$ ) is an indecomposable regular $C$-module of regular length 2 (respectively, regular length 1 ) lying in a tube $T$ in $\Gamma_{C}$ having $n-2$ rays, and $t+1(2 \leq t)$ is the number of objects in $C[N, t]$ which are not in $C$.

2) Every proper convex sub category of $A$ is of polynomial growth.

In particular, we say that the algebra $A$ is 2-tubular if $A=\widetilde{\mathbf{D}_{\mathbf{n}}}[M]$, where $M \in i n d \widetilde{\mathbf{D}_{\mathbf{n}}}$ is regular indecomposable of length 2 lying in a tube $T$ in $\Gamma_{\widetilde{\mathbf{D}_{\mathbf{n}}}}$ having $n-2$ rays.

Proposition 2.1 (1.4 in [P1]). A pg-critical algebra $A$ is derivedequivalent to an algebra given by the following quiver:

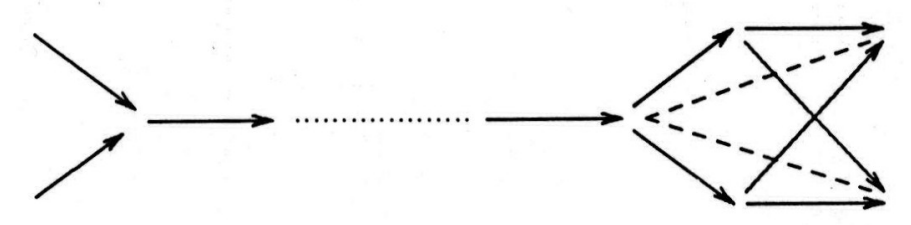

Figure 1.

With the commutative relations, indicated by dotted edges.

Let $A$ be a finite-dimensional $k$-algebra, and $D=H_{o} m_{k}(-, k)$ denote the standard duality on $\bmod A$. The repetitive algebra $\widehat{\mathbf{A}}$ (see $[\mathrm{HW}]$ ) of $A$ is the self-injective, locally finite-dimensional algebra without identity, defined by:

$$
\widehat{\mathbf{A}}=\left(\begin{array}{ccccc}
\ddots & & & & 0 \\
& A & & & \\
& D A & A & & \\
& & D A & A & \\
0 & & & & \ddots
\end{array}\right)
$$


where matrices have only finitely many non-zero entries, addition is the usual addition of matrices, and multiplication is induced from the canonical bimodule structure of $D A=\operatorname{Hom}_{k}(A, k)$ and the zero map $D A \otimes D A \rightarrow 0$.

It was proved in [W] that if $T_{A}$ is a tilting module and $B=E n d T_{A}$, then $\bmod \widehat{\mathbf{A}} \cong \bmod \widehat{B}$, where $\underline{\bmod }$ denote a stable category in the sense of chapter $\bar{X}$ in $[\mathrm{ARS}]$.

The repetitive algebra $\widehat{\mathbf{A}}$ was introduced as the Galois covering (see $[\mathrm{G}]$ ) of the trivial extension $T(A)=A \propto D A$ of $A$ by its minimal injective cogenerator $D A$. Let $\nu$ the Nakayama automorphism of $\widehat{\mathbf{A}}$ and $G=\langle\nu\rangle$. We consider $\widehat{\mathbf{A}}$ as $k$-category, then we have the Galois cover functor: $F: \widehat{\mathbf{A}} \longrightarrow(\widehat{\mathbf{A}} / G)$, where each element of $\widehat{\mathbf{A}}$ corresponds to an orbit. This functor induces the push-down functor $F_{\lambda}: \bmod \widehat{\mathbf{A}} \longrightarrow \bmod (\widehat{\mathbf{A}} / G)$ and pull-up functor $F . \bmod (\widehat{\mathbf{A}} / G) \longrightarrow$ $\bmod \widehat{\mathbf{A}}$, and by 2.2 in $[\mathrm{HW}]$ we know that $T(A) \cong \widehat{\mathbf{A}} / G$.

A $k$-algebra $\widehat{\mathbf{A}}$ is called $\left(\nu_{A}\right)$-exhaustive, when the push-down functor $F_{\lambda}: \bmod \widehat{\mathbf{A}} \longrightarrow \bmod T(A)$ associated to the Galois cover functor $F: \widehat{\mathbf{A}} \longrightarrow T(A)$ is dense.

We say that the $k$-algebra $A$ is of locally finite support, if for each indecomposable projective module $P$, the isomorphism class of the indecomposable projective module $P^{\prime}$ is such that the number of indecomposable module $M$, with $\operatorname{Hom}_{A}(P, M) \neq 0$ and $\operatorname{Hom}_{A}\left(P^{\prime}, M\right) \neq$ 0 is finite.

In particular, in [LDS] it is show that: If $\widehat{A}$ is of locally finite support if and only if the gldim $A$ (global dimension) is strong and finite, that is, the complexes of the derived category has bounded length.

In [LDS] it was proved that if a $k$-algebra $A$ is locally support finite, then $A$ is $\nu_{A}$-exhaustive. Now, the following theorem given by Assem and Skowroński in [AS2], establishes a classification of the repetitive algebra $\widehat{\mathbf{A}}$ which are locally support finite.

Theorem 2.1. Let $A$ be a $k$-algebra. The following conditions are equivalent:

i) $\widehat{\mathbf{A}}$ is tame and exhaustive.

ii) $\widehat{\mathbf{A}}$ is tame and locally support finite.

iii) There exist an algebra $B$ which is either tilted of Dynkin type, 
or representation-infinite tilted of Euclidean type, or tubular, such that $\widehat{\mathbf{A}} \cong \widehat{B}$.

iv) There exist an algebra $\mathcal{C}$ which is either hereditary of Dynkin or Euclidean type, or tubular canonical, such that $A$ and $\mathcal{C}$ are tiltingcotilting equivalent.

v) $\bmod \widehat{\mathbf{A}}$ is cycle-finite.

vi) There exist an algebra $\mathcal{C}$ which is either hereditary of Dynkin

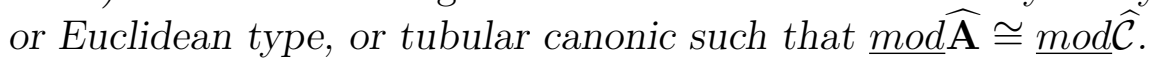

\section{Representation of $T(A)$.}

Let $Q$ be a quiver, $S p$ be a subset of the loops of $Q$, and $\mathcal{R}$ be a set of relations for $Q$. We call the element of $S p$ special loops, the remaining arrows are called ordinary. Let $\mathcal{R}^{S p}:=\left\{x^{2}-x: x \in S p\right\}$, and write $(\overline{\mathcal{R}})$ for the ideal in $k Q /\left(\mathcal{R}^{S p}\right)$ generated b imagen of the element, of $\mathcal{R}$ and denote $J$ the ideal of $k Q /\left(\mathcal{R}^{S p}\right)$ generated by the ordinary arrows.

A triple $(Q, S p, \mathcal{R})$ as above is called clannish (see 2.5 in [C-B]) if the following conditions hold:

1) $(\overline{\mathcal{R}}) \subset J^{2}$

2) for any vertex of $Q$ at most 2 arrows start, and at most 2 arrows stop;

3) for every ordinary arrow $\beta$ there is at most one arrow $\alpha$ with $\alpha \beta \notin$ $\mathcal{R}$, and at most one arrow $\gamma$ with $\beta \gamma \notin \mathcal{R}$.

We consider now the following lemma.

Lemma 3.1. Let $A$ be a 2-tubular $k$-algebra. Then the trivial extension $T(A)$ is tame, and the category $\bmod T(A)$ is equivalent to a category modC , where $\mathcal{C}$ is clannish.

Proof. Let $A$ be a 2-tubular $k$-algebra. By lemma 2.1, we have that $\mathcal{D}^{b}(A) \underset{t}{\cong} \mathcal{D}^{b}(D)$, where $D$ is given by the quiver in figure 1 .

Hence, the ordinary quiver of the trivial extension $T(D)$ is given by: 


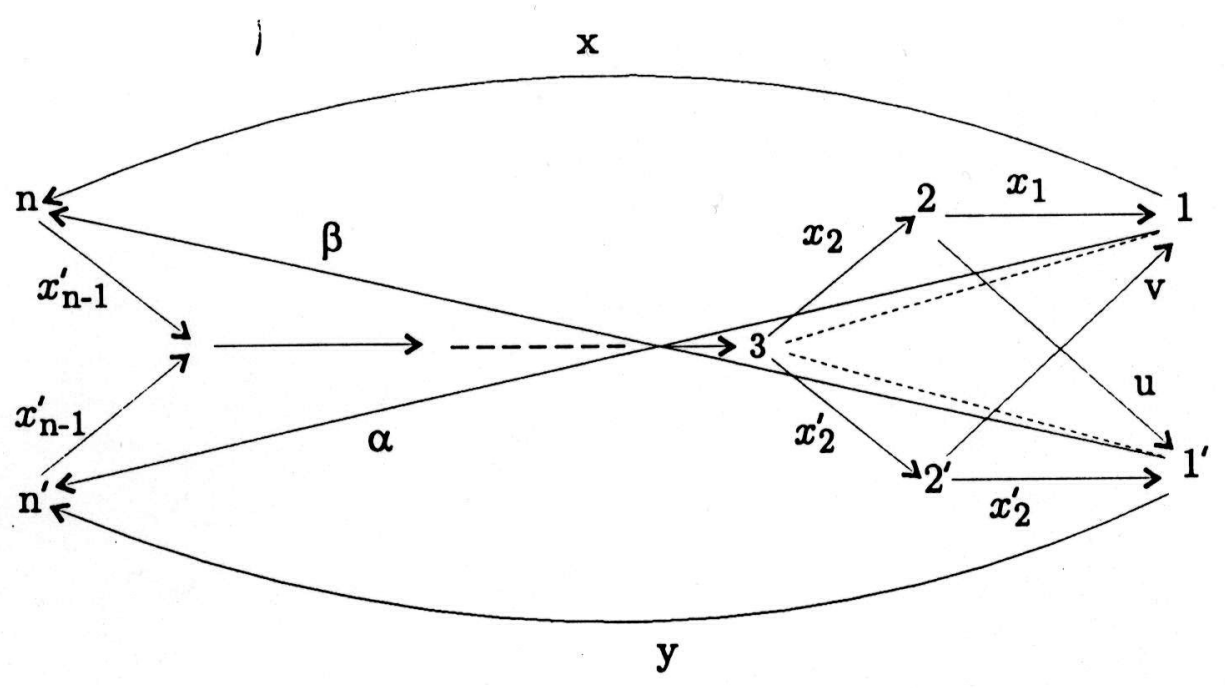

Figure 2 .

with commutativity relations given by: $u \beta=x_{1} x, v \alpha=x^{\prime}{ }_{1} y, x x_{n-1}=$ $\alpha x^{\prime}{ }_{n-1}, x_{2} u=x_{2}{ }_{2} x^{\prime}{ }_{1}, \beta x_{n-1}=y x^{\prime}{ }_{n-1}, x_{2}^{\prime} v=x_{2} x_{1}$ We considered now, the following clannish algebra $\mathcal{C}$, given by the following quiver:

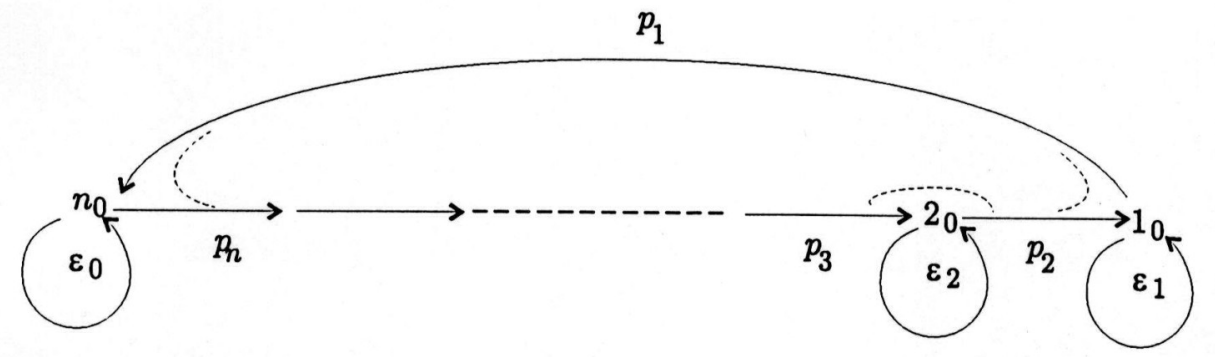

Figure 3. 
where $q_{\varepsilon_{i}}\left(\varepsilon_{i}\right)=\left(\varepsilon_{i}-K_{1}^{i}\right)\left(\varepsilon_{i}-K_{2}^{i}\right)=0$ with $K_{1}^{i} \neq K_{2}^{i} \in k^{*}$, where $i=0,1,2$ and as zero relation we have: $p_{1} p_{n}=p_{3} p_{2}=p_{2} p_{1}=0$.

Now, each module $M \in \bmod \mathcal{C}$, has the form: $M\left(n_{0}\right)=M_{K_{1}^{0}} \oplus M_{K_{2}^{0}}$ where each $M_{K_{i}^{0}}$ is a $k$-vector space associated to the eigenvalue $K_{i}^{0}$ see 2.6 in $[\mathrm{C}-\mathrm{B}]^{2}$.

Then we can defined a functor $F: \operatorname{modT}(D) \longrightarrow \bmod C$ in the following form: Let $X \in \bmod T(D)$

$$
\begin{aligned}
& F(X)\left(i_{0}\right)= \begin{cases}X_{n} \oplus X_{n}^{\prime} & \text { if } i=n \\
X_{2} \oplus X_{2}^{\prime} & \text { if } i=2 \\
X_{1} \oplus X_{1}^{\prime} & \text { if } i=1 \\
X_{i} & \text { if } i \neq\{1,2, n\}\end{cases}
\end{aligned}
$$

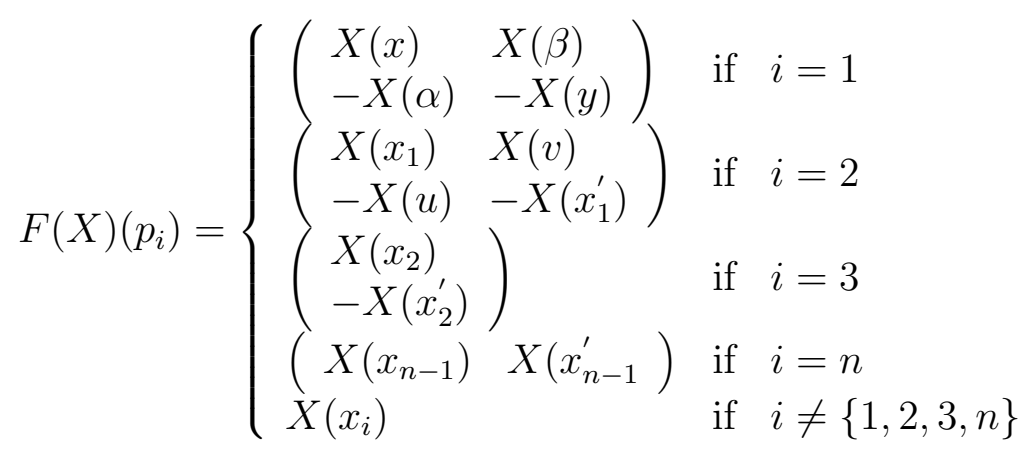

$$
\begin{aligned}
& F(X)\left(\varepsilon_{i}\right)= \begin{cases}\left(\begin{array}{ll}
K_{1}^{1} 1_{x_{1}} & 0 \\
0 & K_{2}^{1} 1_{x_{1}^{\prime}}
\end{array}\right) \text { if } i=1 \\
\left.\begin{array}{ll}
K_{1}^{2} 1_{x_{2}} & 0 \\
0 & K_{2}^{2} 1_{x_{2}^{\prime}}
\end{array}\right) \text { if } i=2 \\
\left.\begin{array}{ll}
K_{1}^{0} 1_{x_{n}} & 0 \\
0 & K_{2}^{0} 1_{x_{n}^{\prime}}
\end{array}\right) \text { if } i=n\end{cases}
\end{aligned}
$$

We define now the functor $G: \bmod C \longrightarrow \bmod T(D)$. Let $M \in$ $\bmod \mathcal{C}$, have that:

$$
p_{i_{0}}=\left(\begin{array}{cc}
M_{11}^{i_{0}} & M_{12}^{i_{0}} \\
M_{21}^{i_{0}} & M_{22}^{i_{0}}
\end{array}\right)
$$

where the $M_{t j}^{i_{0}}: M_{K_{t}^{i_{0}}}=M_{t} \longrightarrow M_{K_{j}^{i_{0}}}=M_{j} \quad i, t, j=1,2$, hence

$$
p_{n}=\left(\begin{array}{ll}
M_{1 n-1}^{n} & M_{2 n-1}^{n}
\end{array}\right), p_{n-1}=\left(\begin{array}{l}
M_{n-1 n-2}^{n-1}
\end{array}\right) \text { and } p_{3}=\left(\begin{array}{c}
M_{31}^{3} \\
M_{32}^{3}
\end{array}\right)
$$


We defined the functor $G$ in the following form: Let $M \in \operatorname{modC}$

$$
\begin{aligned}
& G(M)(x)=\left\{\begin{array}{lll}
M_{2} & \text { if } x=x_{i}, \quad i=1,2, n \\
M_{1} & \text { if } x=x_{i}^{\prime}, \quad i=1,2, n \\
M_{j} & \text { if } x=x_{j}, \quad j \neq\{1,2, n\}
\end{array}\right. \\
& G(M)(x)=M_{22}^{1}\left(p_{1}\right) \quad G(M)\left(x_{1}\right)=M_{22}^{2}\left(p_{2}\right) \\
& G(M)(\alpha)=M_{21}^{1}\left(p_{1}\right) \quad G(M)(u)=M_{21}^{2}\left(p_{2}\right) \\
& G(M)(\beta)=-M_{12}^{1}\left(p_{1}\right) \quad G(M)(v)=-M_{12}^{2}\left(p_{2}\right) \\
& G(M)(y)=-M_{11}^{1}\left(p_{1}\right) \quad G(M)\left(x_{1}^{\prime}\right)=-M_{11}^{2}\left(p_{2}\right) \\
& G(M)\left(x_{2}\right)=M_{32}^{3}\left(p_{3}\right) \quad G(M)\left(x_{n-1}\right)=-M_{23}^{n}\left(p_{n}\right) \\
& G(M)\left(x_{2}^{\prime}\right)=M_{31}^{3}\left(p_{3}\right) \quad G(M)\left(x_{n-1}^{\prime}\right)=M_{13}^{n}\left(p_{3}\right) \\
& G(M)\left(x_{j}\right)=M_{j+1}\left(p_{j}\right) \quad \text { if } \quad j \neq\{1,2,3, n\}
\end{aligned}
$$

Hence, from the relations of Clannish algebra $\mathcal{C}$, is easy verify that this definition of the functor $G$, defined a module in $\bmod T(D)$. By the construction these functors $F$ and $G$ we have that $F \circ G=1_{\operatorname{modT}(D)}$ and $G \circ F=1_{\bmod } \mathcal{C}$. But, is known that an algebra Clannish is tame (see $[\mathrm{C}-\mathrm{B}]$ ), then we have that the trivial extension $T(D)$ is tame. Since, a derived equivalence induces a stable equivalence between the trivials extensions (see $[\mathrm{HW}]$ ), then $\underline{\bmod } T(A) \cong \bmod T(D)$ then by Krause (see $[\mathrm{Kr}]$ ) we have that the trivial extension $T(A)$ is tame.

Proposition 3.1 ( prop. 2 in [DS]). Let $R$ be a locally bounded $k$-category, and $G$ be the group of the $k$-linear automorphism of $R$ acting freely on the objects of $R$. If $R / G$ is tame, then $R$ also is tame.

Theorem 3.1. Let $A$ be a tame concealed algebra, $M$ be an indecomposable module in $\bmod A$, and assume $B=A[M]$ is of tame representation. Then the trivial extension $T(B)$ is tame if and only if the module $M$ is regular.

Proof. Assume $T(B)$ is tame. How, $T(B) \cong \widehat{B} /(\nu)$, then by Proposition 3.1 , we have that $\widehat{B}=\widehat{A[M}]$ is tame. Moreover, by lemma 3 in Ringel [R2] if $A[M]$ is tame, then the module $M$ is regular or preinjective. 
If the module $M$ is preinjective, it is known that $\widehat{A[M}] \cong[\widehat{M]} A$, and $[M] A$ is wild by lemma 3 in $[\mathrm{R} 2]$, hence a full wild subcategory of $[\widehat{M]} A$. Thus, $\widehat{B}$ is wild, which is a contradiction. Therefore, the module $M$ is regular.

Assume $M$ is an indecomposable regular module. We consider two situations :

a) The algebra $A$ is not concealed of type $\widetilde{\mathbf{A}_{\mathbf{n}}}$. Hence, we have that the algebra $B$ is tubular or 2 -tubular (see 2.2 in $[\mathrm{P}]$ ).

If $B$ is tubular, then by theorem 2.1 we have that $\widehat{B}$ is tame and exhautive, therefore the trivial extension $T(B)$ is tame. If $B$ is 2tubular, then by lemma 3.1 we have that the trivial extension $T(B)$ is tame.

b) $A=\widetilde{\mathbf{A}_{\mathbf{n}}}$. We use to the table given by Ringel (see th. 3 in [R2]), we have two situations:

b1) The module $M$ is homogeneus. In this situation we have:

1) $\left(\widetilde{\mathbf{A}_{22}}, 1\right)$; 2) $\left(\widetilde{\mathbf{A}_{23}}, 1\right)$; 3) $\left(\widetilde{\mathbf{A}_{24}}, 1\right)$; 4) $\left(\widetilde{\mathbf{A}_{25}}, 1\right)$; 5) $\left(\widetilde{\mathbf{A}_{26}}, 1\right)$; 6) $\left(\widetilde{\mathbf{A}_{2 q}}, 1\right)$ if $q \geq 7$

7) $\left(\widetilde{\mathbf{A}_{33}}, 1\right)$; 8) $\left(\widetilde{\mathbf{A}_{34}}, 1\right)$; 9) $\left(\widetilde{\mathbf{A}_{35}}, 1\right)$; 10) $\left(\widetilde{\mathbf{A}_{36}}, 1\right)$;

11) $\left(\widehat{\mathbf{A}_{44}}, 1\right)$

In the situation 1 ) to 6 ), these algebras are domestic tubular of type $(2,2, q)$ and that corresponds to the tubular type of $\mathbf{D}_{q+2}$. In the situation 7 ) at 9$)$, this algebras are domestic tubular of type $(2,3,3)$, $(2,3,4)$ and $(2,3,5)$ which corresponds to $\mathbf{E}_{6}, \mathbf{E}_{7}$ and $\mathbf{E}_{8}$ respectively. Now, the situation 10) and 11) is also tubular of type $(2,3,6)(2,4,4)$ corresponding to euclidean type $\widetilde{\mathbf{E}_{8}}$ and $\widetilde{\mathbf{E}_{7}}$ respectively. By theorem 2.1 we have that the trivial extension $T(B)$ is tame.

b2) The module $M$ is not homogeneus. Hence, we have two cases:

b2.1) $\left(\widetilde{\mathbf{A}_{p q}}, p\right)$, where the module $M$ lies in the mouth of tube of rank $p$. Hence, $\left(\widetilde{\mathbf{A}_{p+1 q}}\right)$ which is tubular, therefore the trivial extension $T(B)$ is tame.

b2.2) $\left(\widetilde{\mathbf{A}_{\mathbf{p q}}}, 2 p\right)$, where the module $M$ lies in a tube of rank $p$, and regular of length 2. It is easy to see that the proof of theorem 3 in Ringel [R2] if $\widetilde{\mathbf{A}_{\mathbf{p q}}}[N]$ with module $N$ has regular length 2 there exist a sequence of reflextions which takes $B$ into $\widetilde{\mathbf{A}_{\mathbf{p q}}}[N]$, then $\mathcal{D}^{b}(B) \underset{t}{\text { group }}$ $\cong \mathcal{D}^{b}\left(\widetilde{\mathbf{A}_{\mathbf{p q}}} \widetilde{\mathbf{N}]}\right)$. 
We consider the $C$ algebra, given by the quiver in the following figure:

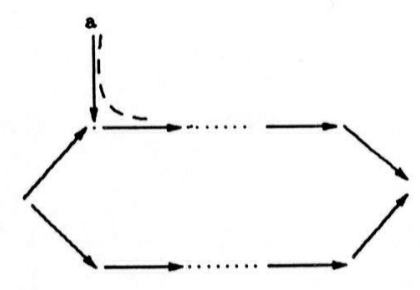

Figure 4.

where the dotted lines is zero relation. We have that $C:=\widetilde{\mathbf{A}_{p-1 q}}[M]$ where the module $M$ is defined by:

$$
M:=\begin{array}{ccccc}
0 & & 1 & 0 & \ldots \\
& & & & \\
& 0 & \ldots & 0
\end{array} \quad \text { that is lies in the mouth the tube of rank }
$$

$p-1$, the algebra $C$ is a tilted algebra of type $\widetilde{\mathbf{A}_{p q}}$ then there exist a functor $\rho: \mathcal{D}^{b}(C) \longrightarrow \mathcal{D}^{b}\left(\widetilde{\mathbf{A}_{p q}}\right)$, that give a triangular equivalence such that $\rho(M)$ lyies in the tube of rank $p$, and is a regular of lenght 2.

We consider now the algebra $D$ defined by the following quiver:

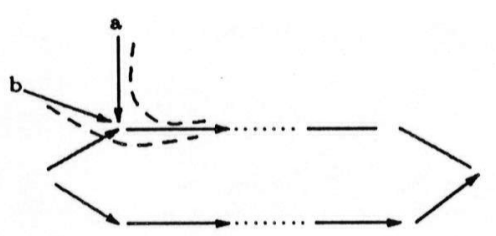

Figure 5 . 
Here the module $M$ is the same as before. Then, by Barot-Lenzing (see $[\mathrm{BL}])$, we have that: $\mathcal{D}^{b}(D) \cong \mathcal{D}^{b}\left(\left(\widetilde{\mathbf{A}_{p q}}\right)[\rho(M)]\right)$, where $\widetilde{\mathbf{A}_{p q}}[\rho(M)]$ is a one-point extension by module $\rho(M)$ of regular length 2 , then $\mathcal{D}^{b}(B) \cong \mathcal{D}^{b}\left(\left(\widetilde{\mathbf{A}_{p q}}\right)[\rho(M)]\right)$. Using the similar construction as the lemma 3.1 with clannish algebras is not dificult to see that $\bmod D$ is equivalent to a clannish algebra $E$ given by the following quiver:

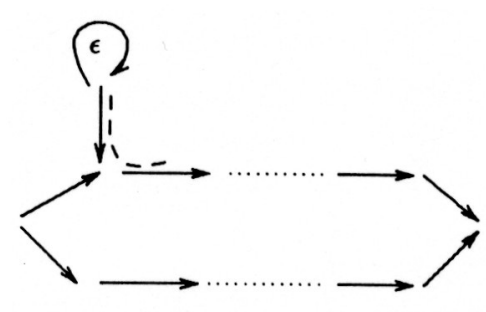

Figure 6.

Where $\epsilon$ is special loop. By Geiss and De la Peña (see 4.4 in [GEP]) the trivial extension $T(E)$ is tame, thus $T(D)$ is tame. Since $\mathcal{D}^{b}(D) \cong \mathcal{D}^{b}(B)$, therefore $\underline{\bmod } T(D) \cong \underline{\bmod } T(B)$, then by $[\mathrm{Kr}]$ we have that $T(B)$ is tame.

Before to state the next result, we consider $A \cong k Q / I$ be an algebra, where $Q$ is a quiver without oriented cycles. Let $\chi_{A}: \mathbf{Z}^{Q_{0}} \longrightarrow \mathbf{Z}$ and $q_{A}: \mathbf{Z}^{Q_{0}} \longrightarrow \mathbf{Z}$ be the quadratic forms defined by:

$$
\begin{aligned}
& \chi_{A}(v)=\sum_{s=0}^{\infty} \sum_{i, j \in Q_{0}}(-1)^{s} \operatorname{Ext}_{A}^{s}\left(S_{i}, S_{j}\right) v_{i} v_{j} \\
& q_{A}(v)=\sum_{i \in Q} v_{i}^{2}-\sum_{(i \rightarrow j) \in Q_{1}} v_{i} v_{j}+\sum_{i, j \in Q_{0}} r(i, j) v_{i} v_{j}
\end{aligned}
$$

where $v=\left(v_{1}, \ldots, v_{n}\right), r(i, j)=\operatorname{dim}_{k} e_{j}(I /(I J+J I)) e_{i}$, and $J$ is the ideal generated by arrows of the quiver $Q$. The quadratic form $\chi_{A}$ is called the Euler form of the algebra $A$, and $q_{A}$ is called the Tits form. Its know that if $\operatorname{gldim} A \leq 2$, then $\chi_{A}=q_{A}$. 
As the consequence of our theorem 3.1, we have the following corollary.

Corollary 3.1. Let $A \cong k Q / I$, where $Q$ is a tree, such that Euler form $\chi_{A}$ is non-negative and corank $\chi_{A} \leq 2$. Then the tivial extension $T(A)$ is tame.

Proof. By Barot-De la Peña (see $[\mathrm{BP}]$ ) we have that the algebra $A$ is domestic tubular, tubular or 2-tubular. Therefore, by the above theorem we have that the trivial extension $T(A)$ is tame.

\section{References}

[A1] I. Assem. Tilted Algebras of type $\mathbf{A}_{\mathbf{n}}$. Communications in Algebra, (19); 2121-2139, (1982).

[AS2] I. Assem and A. Skowroński. On tame repetitive algebra. Fundamenta Mathematicae. 142; 59-84, (1993).

[ARS] M. Auslander, I. Reiten and S. Smalø. Representation theory of Artin algebras. Cambridge Studies in Advanced Mathematics 36, (1995).

[BL] M. Barot and H. Lenzing. One-point extensions and derived equivalence. to appear.

[BP] M. Barot and J. A. de la Peña. Derived tubular strongly simply connected algebras. In Proceeding Am. Math. Soc. 127 N 3, 647-655, (1999).

[C-B] W.W. Crawley-Boevey. Funtorial filtration II: Clans and the Gelfand problem. J. London Math. Soc. 40, 9-30, (1989).

[G] P. Gabriel. Unzerlegbare darstellungen I. Manuscripta Math. 6; 71-103. (1972). 
[GEP] C. Geiss and J. A. de la Peña. Auslander-Reiten Components for Clans. To appear.

[DS] P. Dowbor and A. Skowroński. Galois corering of tame algebras. Arch. Math. 44; 522-529, (1985).

[H] D. Happel. On the derived category of a finite-dimensional algebras. Comment. Math. Helv. 62; 339-389, (1987).

[HW] D. Hughes and J. Waschbusch. Trivial extension of tilted algebras. Proc. London Math. Soc. 46; 347-364, (1983).

[Kr] H. Krause. Stable module categories and their representation type. Preprint Bielefeld. (1996).

[LDS] H. Lenzing, P. Dowbor and A. Skowroński. Galois covering of algebras by locally suppot-finite categories. Proceeding Letures Notes in Math. 1171, Springer (1986).

[NS] R. Norenberg and A. Skowroński. Tame minimal nonpolinomial growth strongly connected algebras. Proc. ICRA VII. CMS-AMS conference Proceeding Vol. 18; 519-538, (1996).

[N] C. Novoa-Bustos. Tipo de representação e quiver ordinário do bimodulo DA. Tese de doutorado IME USP. (1999).

[P1] J.A. de la Peña. Algebras whose derived category is tame. Proceediings AMS-conference, Seattle (1997).

[P] J.A. de la Peña. On the representation type of one point extension of tame concealed algebras. Manuscripta Math., 61; 183194, (1988).

[R] C. M. Ringel. Tame algebras and quadratic form. Lectures Notes in Math. 1099, Springer,(1984).

[R2] C. M. Ringel. Tame algebras, on algorithms for solving vector space problems II. Proceedings Lecture Notes in Math. 831, Springer (1980). 
[W] T. Wakamatsu. Stable equivalence between universal covers of trivial extension self-injective algebras. Tsukuba J. Math., 9; 299-316, (1985).

Received : July 2001.

\section{Cristián Novoa Bustos}

Dept. Matemática e Física

Universidade Católica de Goiás

Av. Universitria 1440 / st. Universitario

CEP 74000-000 Goiânia-Go

Brasil

cristiannovoa@netscape.net

and

\section{José Antonio de la Peña}

Instituto de Matemática

Circuito Exterior

Ciudad Universitaria

México 04510, D. F.

México

jap@penelope.matem.unam.mx 